\title{
Baicalin inhibits proliferation and promotes apoptosis of vascular smooth muscle cells by regulating the MEG3/p53 pathway following treatment with ox-LDL
}

\author{
YUN LIU $^{1 *}$, LIANQUN JIA ${ }^{2 *}$, DONGYU MIN ${ }^{3}$, YI XU ${ }^{1}$, JINQUAN ZHU $^{1}$ and ZENGXIAN SUN ${ }^{1}$ \\ ${ }^{1}$ Department of Pharmacy, The First People's Hospital of Lianyungang, Lianyungang, Jiangsu 222002; ${ }^{2}$ Key Laboratory of \\ Ministry of Education for Traditional Chinese Medicine Viscera State Theory and Applications, Liaoning University of \\ Traditional Chinese Medicine, Shenyang, Liaoning 110847; ${ }^{3}$ Traditional Chinese Medicine Experimental Center, \\ Affiliated Hospital of Liaoning University of Traditional Chinese Medicine, Shenyang, Liaoning 110032, P.R. China
}

Received July 11, 2018; Accepted November 26, 2018

DOI: $10.3892 / \mathrm{ijmm} .2018 .4009$

\begin{abstract}
Atherosclerosis (AS) is a systemic disease associated with lipid metabolic disorders and abnormal proliferation of smooth muscle cells. Baicalin is a flavonoid compound isolated from the dry roots of Scutellaria baicalensis Georgi and exerts anti-proliferative effects in various types of cells. However, the effect of baicalin on AS remains unclear. In the present study, serum samples were collected from patients with AS and an in vitro model of AS was established using oxidized low-density lipoprotein (ox-LDL)-treated human aorta vascular smooth muscle cells (HA-VSMCs). The siRNA transfection and overexpression efficiency of endogenous maternally expressed gene 3 (MEG3) and the expression level of MEG3 were analyzed by reverse transcription-quantitative polymerase chain reaction (RT-qPCR). The effects of alterations in expression levels of MEG3 were assessed by MTT assay, bromodeoxyuridine incorporation assay, 5-ethynyl-2'-deoxyuridine staining, wound healing assay, immunofluorescence and western blotting in HA-VSMCs. qPCR indicated that the expression of MEG3 was reduced in serum samples from patients with AS and ox-LDL-treated HA-VSMCs, compared with serum samples from healthy patients and untreated HA-VSMCs, respectively. Further experiments indicated that ox-LDL-induced decrease of MEG3 expression was reversed by treatment with baicalin in a concentration-dependent manner. Following treatment with ox-LDL, decreased expression of MEG3 promoted proliferation
\end{abstract}

Correspondence to: Dr Yun Liu, Department of Pharmacy, The First People's Hospital of Lianyungang, 182 Tongguan North Road, Lianyungang, Jiangsu 222002, P.R. China

E-mail: yunliu211315@163.com

*Contributed equally

Key words: atherosclerosis, maternally expressed gene 3, human aorta vascular smooth muscle cells, p53 and migration, and suppressed apoptosis in HA-VSMCs. Furthermore, treatment with baicalin reversed these effects on proliferation and apoptosis in ox-LDL-treated HA-VSMCs. The current study indicated that downregulated expression of MEG3 increased cell cycle-associated protein expression. However, treatment with baicalin inhibited the expression of cell-cycle associated proteins in HA-VSMCs with MEG3 knockdown. In addition, baicalin activated the p53 signaling pathway and promoted the expression and transport of p53 from the cytoplasm to nucleus following MEG3 knockdown in ox-LDL-treated HA-VSMCs. Baicalin inhibited proliferation and promoted apoptosis by regulating the expression of MEG3/p53, indicating that baicalin may serve a role in AS by activating the MEG3/p53 signaling pathway. The present study suggested a potential mechanism underlying the protective role of baicalin in the in vitro model of AS, and these results may be used to develop novel therapeutic approaches for the affected patients.

\section{Introduction}

Atherosclerosis (AS) is a common and detrimental disease characterized by lipid deposition in arterial intima, abnormal proliferation of vascular smooth muscle cells (VSMCs) and connective tissue hyperplasia, leading to intimal focal fibrous thickening and atherosclerotic plaque formation. Hardening and narrowing of the arteries are frequently observed among patients with AS. AS may lead to cardiovascular, cerebrovascular and thromboembolic diseases. Proliferation and angiogenesis of VSMCs are important biological processes in the development of AS. Therefore, inhibition of excessive proliferation and induction of apoptosis of VSMCs could be an effective method for the treatment and control of AS (1). Although there were a number of studies investigating the etiology of AS (2-4), the exact mechanism remains to be elucidated.

Long noncoding (lnc)RNAs are a group of RNA molecules that lack protein-coding function and are $>200$ nucleotides in length (5). It has been demonstrated that lncRNAs are involved in the regulation of various biological processes in cells, 
including proliferation, migration, apoptosis and differentiation (6). Although the function and mechanism of lncRNAs remain to be completely elucidated, previously published studies indicated that lncRNAs are closely associated with AS and development of other cardiovascular diseases. LncRNAs associated with AS have been studied by researchers due to their regulatory roles in lipid metabolism, proliferation, migration, apoptosis, adhesion and inflammatory response (7). It has been previously demonstrated that the expression levels of long intergenic noncoding (linc)RNA-p21 decreased in AS-prone apolipoprotein E (ApoE)-deficient atherosclerotic plaques and lincRNA-p21 repressed cell proliferation and induced apoptosis of VSMCs by enhancing transcriptional activity of p53 (2). Smooth muscle induced lncRNA, enhancer of proliferation and metastasis associated lung adenocarcinoma transcript 1 have been reported to be involved in controlling vascular cell proliferation (8), endothelial cell (EC) sprouting (9) and phenotypic transition (10). Long intergenic non-protein coding RNA 599 silencing contributed to EC and VSMC dysfunction, and aggravated AS (11). Maternally expressed gene 3 (MEG3) is an imprinted gene with a length of $\sim 1.6 \mathrm{~kb}$, and its transcriptional deficiency may induce the occurrence and development of multiple tumors, including non-small cell lung cancer (12), gastric cancer (13) and liver cancer (14). Our recent study indicated that the expression of MEG3 was decreased in pulmonary arteries of patients with pulmonary hypertension resulting in increased human pulmonary artery smooth muscle cell proliferation and migration through the p53 signaling pathway (15). However, the function and mechanism of MEG3 in AS remain to be elucidated, and, therefore, it is necessary to further investigate the molecular mechanism of MEG3 in the development of AS.

Scutellaria baicalensis Georgi is a commonly used herbal medicine, which exhibits a variety of therapeutic effects in traditional Chinese Medicine formulations (16). Baicalin (Fig. 1) (17), the main active component of Scutellaria baicalensis Georgi, is a flavonoid compound extracted from the dry roots exhibiting biological activity (18). Previous studies indicated that baicalin may induce numerous pharmacological effects, including anti-oxidative (19), antitumor (20), anti-inflammatory (21) and antiproliferative (17) functions. Baicalin inhibited the activation of nuclear factor- $\kappa \mathrm{B}$, decreased the expression of pro-inflammatory mediators and prevented renal dysfunction in ApoE knock out mice on high-cholesterol diets, which served an important role in the prevention of AS $(22,23)$. A number of studies reported that $\mathrm{p} 53$ serves an important role in the pathogenesis of AS $(2,24,25)$. Furthermore, p53 regulates cell cycle and apoptosis $(15,25)$. $\mathrm{Wu}$ et al (2) hypothesized that lincRNA-p21 may regulate vascular smooth muscle cell proliferation and apoptosis by enhancing the activity of p53 in AS (2). Our previous studies suggested that MEG3 may increase the activity of p53 in pulmonary artery smooth muscle cells (15). The current results indicated that the expression levels of MEG3 decreased in serum samples from patients with AS and oxidized low-density lipoprotein (ox-LDL)-stimulated human aorta vascular smooth muscle cells (HA-VSMCs) compared with the control samples. Treatment with baicalin promoted the expression of MEG3 and inhibited the proliferation of HA-VSMCs induced by MEG3 knockdown. MEG3 knockdown increased the expression of proliferating cell nuclear antigen (PCNA), cyclin A and E. However, following treatment of HA-VSMCs with different concentrations baicalin, expression of PCNA, cyclin A and E was inhibited in cells with MEG3 knockdown. The p53 signaling pathway components are expressed in the nucleus under normal conditions (26); the expression of p53 was detected in the cytoplasm after MEG3 knockdown. When HA-VSMCs were treated with different concentrations of baicalin, p53 expression was detected in the nucleus. In addition, the protein expression level of p53 decreased compared with the NC group after MEG3 knockdown. Baicalin could increase p53 protein expression after MEG3 knockdown in ox-LDL-treated HA-VSMCs. In conclusion, the current study aimed to further investigate the underlying roles and molecular basics of baicalin and MEG3/p53 in the progression to AS, implicating the potential values of baicalin and MEG3 in AS therapy.

\section{Materials and methods}

Reagents and antibodies. Baicalin (purity, >99.0\%; Sigma-Aldrich; Merck KGaA, Darmstadt, Germany) was dissolved in dimethylsulfoxide. The antibodies against PCNA (cat. no. 10205-2-AP; 1:1,000), cyclin A (cat. no. 13295-1-AP; 1:2,000), cyclin E (cat. no. 11554-1-AP; 1:2,000) and $\beta$-actin (cat.no. 60008-1-1g; 1:5,000) were purchased from ProteinTech Group, Inc. (Chicago, IL, USA). CycleTEST ${ }^{\mathrm{Tm}}$ PLUS DNA Reagent kit was obtained from BD Biosciences (Franklin Lakes, NJ, USA). The bromodeoxyuridine (BrdU) proliferation kit was purchased from EMD Millipore (Billerica, MA, USA). Ox-LDL, VicFemto enhanced chemiluminesence (ECL) kit and PBS were purchased from Nanjing KeyGen Biotech Co., Ltd. (Nanjing, China).

Clinical samples. The present study was approved by the Ethics Committee for the use of human samples of the First People's Hospital of Lianyungang, which was in accordance with the code of ethics of the Declaration of Helsinki developed by the World Medical Association. Each individual provided written informed consent prior to their participation. Serum samples were collected from 40 patients with AS (age, $60 \pm 10$ years; 18 male and 22 female) and 40 healthy volunteers (age, 58 \pm 9 years; 21 male and 19 female) from the Department of Cardiovascular Medicine, the First People's Hospital of Lianyungang (Lianyungang, China) between January 2015 and January 2016.

Clinical inclusion and exclusion criteria. The AS group included patients with $>80 \%$ coronary artery stenosis confirmed by coronary angiogram. The control group included patients without clinically significant coronary artery occlusion diagnosed by coronary angiogram. Patients with vascular diseases, including hypertension and type I/II diabetes were excluded. Total RNA was extracted from peripheral blood monocytes of patients and controls.

Cell culture. HA-VSMCs were obtained from American Type Culture Collection (Manassas, VA, USA) and cultured in F-12K medium (American Type Culture Collection) 
<smiles>O=C(O)[C@H]1O[C@@H](Oc2cc3oc(-c4ccccc4)cc(=O)c3c(O)c2O)[C@H](O)[C@@H](O)[C@@H]1O</smiles>

Figure 1. Chemical structure of baicalin.

containing $10 \%$ heat-inactivated fetal bovine serum (FBS; Gibco; Thermo Fisher Scientific, Inc., Waltham, MA, USA). Other nutrients added to the HA-VSMC culture were based on previously published literature (27). Cells were incubated at $37^{\circ} \mathrm{C}$ with $5 \% \mathrm{CO}_{2}$.

Cell transfection and treatment. The sequences of siRNA targeting lncRNA MEG3 and scrambled negative control siRNA (si-NC) were designed and synthesized by GenePharma (Shanghai GenePharma Co., Ltd., Shanghai, China) according to the previously published protocol (15). pcDNA-MEG3 and empty vector (pcDNA) were synthesized by GenePharma (Shanghai GenePharma Co., Ltd.). At 60\% confluence, HA-VSMCs were cultured in Dulbecco's modified Eagle's medium (Gibco; Thermo Fisher Scientific, Inc.) at $37^{\circ} \mathrm{C}$ with $5 \% \mathrm{CO}_{2}$ for $24 \mathrm{~h}$. HA-VSMCs were transfected with si-NC, si-MEG3 at a final concentration of $20 \mathrm{nmol} / \mathrm{l}$ in accordance with the manufacturer's protocol. Furthermore, HA-VSMCs were transfected with pcDNA-MEG3 at a final concentration of $2 \mathrm{mg} / \mathrm{l}$ using. The following sequences were used: si-lncRNA MEG3, 5'-GGGCTTCTGGAATGAGCAT-3'; si-NC, 5'-UUC UCCGAACGUGUCACGUTT-3' (15). HA-VSMCs were transfected using Lipofectamine ${ }^{\circledR} 2000$ on 6 -well plates (Invitrogen; Thermo Fisher Scientific, Inc.) according to the manufacturer's protocol. Cells were harvested for the subsequent experiments $24 \mathrm{~h}$ after transfection.

DNA BrdU incorporation assay. Cell proliferation analysis was performed with BrdU. Transfected and untransfected cells were seeded into 96-well plates at a density of $1 \times 10^{4}$ cells/well and treated with control (no treatment) and different concentrations of ox-LDL $(25,50$ or $75 \mu \mathrm{g} / \mathrm{ml})$ and baicalin $(5,10$ or $20 \mu \mathrm{mol} / \mathrm{l}$ ) in DMEM containing $2 \% \mathrm{FBS}$ at $37^{\circ} \mathrm{C}$ with $5 \% \mathrm{CO}_{2}$ and $\mathrm{BrdU}$ was added to the proliferating cells $2-24 \mathrm{~h}$ before the end of the test reagent incubation. The following steps were performed according to the manufacturer's protocol and as previously described (28). Finally, the absorbance was measured at a dual wavelength of $450 / 550 \mathrm{~nm}$.

Reverse transcription-quantitative polymerase chain reaction $(R T-q P C R)$. Total RNA was isolated from serum samples and HA-VSMCs using the TRIzol reagent according to the manufacturer's protocol (Thermo Fisher Scientific, Inc.). RT reaction and qPCR were performed as previously described (15). cDNA was reverse transcribed from $100 \mathrm{ng}$ of total RNA using $4 \mu \mathrm{l}$ of miScript HiSpec buffer, $2 \mu 1$ of Nucleics Mix, $2 \mu 1$ of miScript Reverse Transcriptase mix (Qiagen GmbH, Hilden,
Germany) and sterilized distilled water up to a total volume of $20 \mu 1$. The RT reaction was performed at $37^{\circ} \mathrm{C}$ for $60 \mathrm{~min}$ followed by heat inactivation at $95^{\circ} \mathrm{C}$ for $5 \mathrm{~min}$. The qPCR reactions were performed in triplicate using 7500 Fast Real-Time PCR System (Applied Biosystems; Thermo Fisher Scientific, Inc.). Reaction mixtures had a total volume of $25 \mu 1$, including $2 \mu \mathrm{l}$ of cDNA, $12.5 \mu \mathrm{l}$ of QuantiTect SYBR-Green PCR master mix (Qiagen $\mathrm{GmbH}$ ), $2.5 \mu \mathrm{l}$ of universal primers (Qiagen $\mathrm{GmbH}$ ), $2.5 \mu \mathrm{l}$ of MEG3 or GAPDH-specific primers and $5.5 \mu \mathrm{l}$ of nuclease-free water. Reactions were incubated in 96-well optical plates (Applied Biosystems; Thermo Fisher Scientific, Inc.) using the following thermocycling conditions: Initial denaturation at $95^{\circ} \mathrm{C}$ for $10 \mathrm{~min} ; 40$ cycles of $95^{\circ} \mathrm{C}$ for $10 \mathrm{sec}$ and $60^{\circ} \mathrm{C}$ for $30 \mathrm{sec}$. At the end of the PCR cycles, melting curve analysis was performed to validate the specific generation of the expected PCR products. Primers were designed using Applied Biosystems Primer Express 3.0 (Applied Biosystems; Thermo Fisher Scientific, Inc). GAPDH was used as internal reference and the $2^{-\Delta \Delta C q}$ method was used to calculate the expression of IncRNA MEG3 in samples from serum and cells (29). The following primer sequences were used for the qPCR: MEG3: 5'-CTGCCCATCTACACCTC ACG-3' (forward) and 5'-CTCTCCGCCGTCTGCGCTAGG GGCT-3' (reverse); GAPDH: 5'-GAAGGTGAAGGTCGG AGTC-3' (forward) and 5'-GAAGATGGTGATGGGATTTC-3' (reverse).

Western blotting. A total of $24 \mathrm{~h}$ after transfection, cells were washed 3 times with PBS and incubated with radioimmunoprecipitation assay lysis buffer (cat. no. KGP702-100; Nanjing KeyGen Biotech Co., Ltd.) for $30 \mathrm{~min}$ on ice. After $30 \mathrm{~min}$ of cell lysis, cell lysates were sonicated with $20 \mathrm{KHz}$ frequency on ice for $1 \mathrm{~min}$ and centrifuged at $14,000 \mathrm{xg}$ for $15 \mathrm{~min}$ at $4^{\circ} \mathrm{C}$, and the protein concentration was quantified using bicinchoninic acid protein concentration kit (Nanjing KeyGen Biotech Co., Ltd.). Samples containing $20 \mu \mathrm{g}$ total protein were analyzed using SDS-PAGE (10\% gel) using an electrophoresis instrument (Bio-Rad Laboratories, Inc., Hercules, CA, USA), and western blotting was performed according to the previously published protocol (28). Antibodies against PCNA, cyclin A and E, p53 and $\beta$-actin were used overnight at $4{ }^{\circ} \mathrm{C}$ and washed 6 times with tris buffered saline with Tween-20 (TBST) at room temperature (5 $\mathrm{min} /$ wash). Subsequently, the membrane was treated with horseradish peroxidase labeled goat-anti-rabbit immunoglobulin $\mathrm{G}$ (IgG) secondary antibody (cat. no. VA002; 1:5,000; Xuzhou VICMED Biological Technology Co., Ltd., Xuzhou, China; www.vicmed.cn) or goat-anti-mouse IgG secondary antibody (cat. no. VA001; 1:5,000; Xuzhou VICMED Biological Technology Co., Ltd.), shaken and incubated at room temperature for $1 \mathrm{~h}$. Following washing with TBST 6 times at room temperature $(5 \mathrm{~min} /$ wash), the membranes were incubated with ECL solution for $5 \mathrm{~min}$. Bands were imaged using VersaDoc ${ }^{\mathrm{TM}}$ MP 4000 (Bio-Rad Laboratories, Inc.) and analyzed by using PDQuest Advanced 2D analysis software (version 8.0, Bio-Rad Laboratories, Inc.).

Flow cytometry analysis of cell cycle. The transfected HA-VSMCs were cultured in 6-well plates. To perform cell cycle analysis, the cells were digested with trypsin and fixed 
with $70 \%$ ethanol for $24 \mathrm{~h}$ at $4^{\circ} \mathrm{C}$. The cells were stained according to the manufacturer's protocol of CycleTEST PLUS DNA Reagent kit (BD Biosciences). The fixed cells were washed 3 times with PBS and centrifuged at $300 \mathrm{x}$ g for $5 \mathrm{~min}$ in room temperature. The supernatant was subsequently discarded. Cells were incubated with propidium iodide (PI) stain buffer for $10 \mathrm{~min}$ and subsequently treated with $200 \mu \mathrm{l}$ of PBS, $200 \mu \mathrm{l}$ of RNase A and $200 \mu \mathrm{l}$ of PI for $10 \mathrm{~min}$ at $4^{\circ} \mathrm{C}$ in the dark. Finally, the cells were screened by 400 -mesh sieves and analyzed by flow cytometry (FACSCanto II; BD Biosciences). The results obtained were analyzed using the ModFit software (version 4.1; Verity Software House, Inc., Topsham, ME, USA).

Wound healing assay. For the wound healing assay, the following groups of HA-VSMCs were cultured in 6-wellplates until $60 \%$ confluence: control, si-NC, si-lncRNA MEG3, si-lncRNA MEG3 + baicalin (5 $\mu \mathrm{mol} / 1)$, si-lncRNA MEG3 + baicalin (10 $\mu \mathrm{mol} / \mathrm{l})$, si-lncRNA MEG3 + baicalin (20 $\mu \mathrm{mol} / \mathrm{l})$. Cells were scratched with pipette tips and washed three times with PBS (15). Images of scratch areas were captured at 0 and $24 \mathrm{~h}$. Each experiment was repeated five times independently.

Mitochondrial depolarization assay. The potential of mitochondrial membrane was analyzed as previously described using JC-1 staining (30). Mitochondrial depolarization is demonstrated by increased green/red fluorescence intensity ratio. When mitochondrial membrane potential level is low, green fluorescence is primarily observed. When the mitochondrial membrane potential increases, a polymer is formed and red fluorescence is observed (31). Mitochondrial membrane potentials were detected using a fluorescent microscope with $488 \mathrm{~nm}$ excitation wavelength.

5-Ethynyl-2'-deoxyuridine (EdU) staining. Cell proliferation was analyzed using kFluor647 Click-iT EdU kit (Nanjing KeyGen Biotech Co., Ltd.) according to the manufacturer's protocol. HA-VSMCs were seeded into 96-well cell culture plates at a density of $1 \times 10^{4}$ cells/well. Following transfection and treatment with baicalin, HA-VSMCs were incubated with $10 \mu \mathrm{mol} / 1 \mathrm{EdU}$ for $4 \mathrm{~h}$ at $37^{\circ} \mathrm{C}$. Cells were permeabilized for 20 min with $0.5 \%$ Triton X-100 after fixing with $4 \%$ formaldehyde for $30 \mathrm{~min}$ at room temperature. Cells in each well were washed three times with $0.1 \mathrm{ml} 3 \%$ bovine serum albumin (cat. no. VIC018; Xuzhou VICMED Biological Technology Co., Ltd.) in PBS and subsequently incubated with $1 \mathrm{X}$ Click-iT EdU reaction buffer at room temperature for $30 \mathrm{~min}$ in the dark. HA-VSMC nuclei stained by Hoechst were used to count cells and visualization was performed using a fluorescence microscope as described below (Nikon Corporation, Tokyo, Japan).

Hoechst staining. Treated HA-VSMCs were cultured on 24-well cell culture plates. Subsequently, $1 \mu 1$ of Hoechst 33342 (5 mg/ml; Sigma-Aldrich; Merck KGaA) in $200 \mu \mathrm{l}$ of PBS was added to HA-VSMCs and incubated for $20 \mathrm{~min}$ in room temperature. The stained cells were washed three times with PBS, and cell morphology was observed under a fluorescence microscope.
Immunofluorescence. HA-VSMCs were fixed with 4\% paraformaldehyde for $15 \mathrm{~min}$ at room temperature, and washed in cold PBS 3 times for $5 \mathrm{~min}$. Permeabilization was performed in 0.4\% Triton X-100 (Sigma-Aldrich; Merck KGaA) for 10 min at room temperature. For immunofluorescence staining, the experiment was conducted as previously described (15).

Cell viability assay. Cell viability was assayed by the MTT assay, as previously described (28). HA-VSMCs were cultured in 96-well microtitration plates and then the cells were subjected to growth arrest in DMEM without serum for $24 \mathrm{~h}$. Following transfection and treatment, HA-VSMCs were incubated for $4 \mathrm{~h}$ in a medium containing $0.5 \% \mathrm{MTT}$. Thereafter, the supernatant was removed, and dimethylsulfoxide ( $150 \mu \mathrm{l} /$ well) was added. The plates were then agitated on a plate shaker for $10 \mathrm{~min}$ at room temperature. The absorbance was measured at a wavelength of $490 \mathrm{~nm}$ in a spectrophotometer.

Statistical analysis. Data are presented as the mean \pm standard error of the mean. Comparisons between two groups were performed using unpaired Student's t-test and three or more groups were compared using one-way analysis of variance, followed by Dunnett's test. $\mathrm{P}<0.05$ was considered to indicate a statistically significant difference.

\section{Results}

Expression level of MEG3 decreases in serum samples from patients with AS and ox-LDL-treated HA-VSMCs, and baicalin increases the expression levels of MEG3 in ox-LDL-treated $H A-V S M C s$. The current study determined the expression levels of MEG3 in serum samples from patients with AS and HA-VSMCs treated with ox-LDL. RT-qPCR assay indicated that the expression level of MEG3 significantly decreased in patients with AS compared with healthy controls (Fig. 2A). A previous study reported that ox-LDL serves an important role in the progression of AS (32). Ox-LDL promotes osteogenic differentiation and proliferation of VSMCs (33), and has been widely used to induce a model of AS $(27,32,33)$. Consistent with these results, the current study indicated that ox-LDL promoted cell proliferation in a concentration dependent manner (Fig. 2B and C). In addition, the expression levels of MEG3 were detected in HA-VSMCs after treatment with ox-LDL. The expression of MEG3 decreased following stimulation with ox-LDL. The results indicated that the expression level of MEG3 decreased in a concentration-dependent manner. The greatest inhibition of MEG3 expression was achieved using a dose of $75 \mu \mathrm{g} / \mathrm{ml}$ ox-LDL, and, therefore, this dose was used in subsequent experiments (Fig. 2D). These results indicated that MEG3 may be a mediator in AS progression. HA-VSMCs treated with ox-LDL were used as an in vitro model of AS and subsequently treated with baicalin. The present study aimed to determine whether different concentrations of baicalin affected the expression of MEG3. Data indicated that different concentrations of baicalin did not affect the expression of MEG3 in the absence of oxLDL treatment (Fig. 2E), suggesting that this drug did not affect the expression of MEG3 in normal cells. However, in ox-LDL-treated HA-VSMCs, incubation with ox-LDL resulted in a significant decrease in MEG3 expression 
A

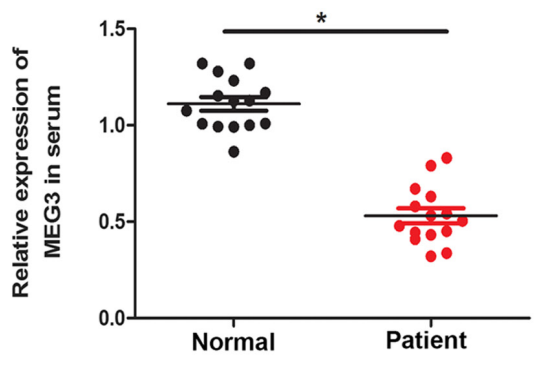

C
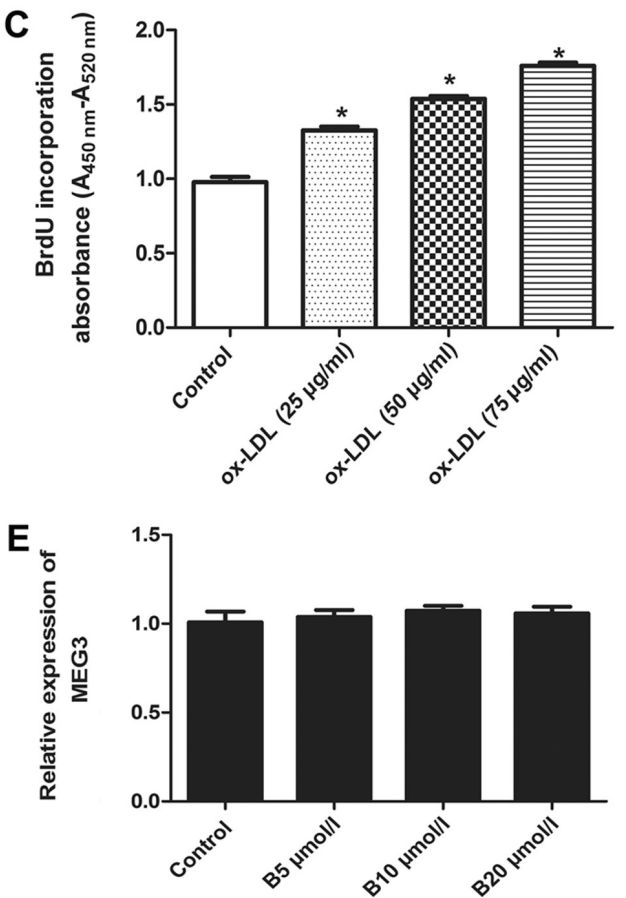

B

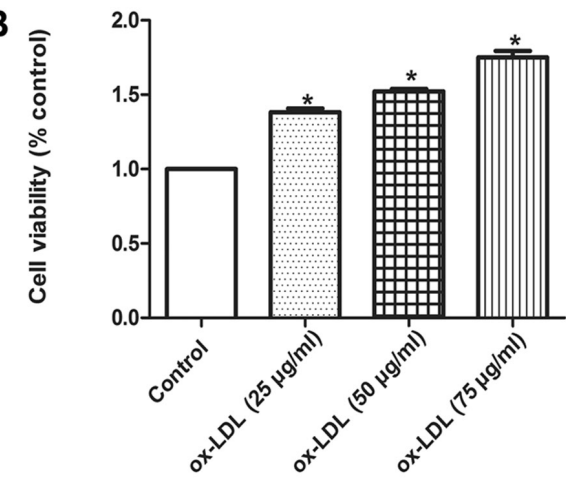

D
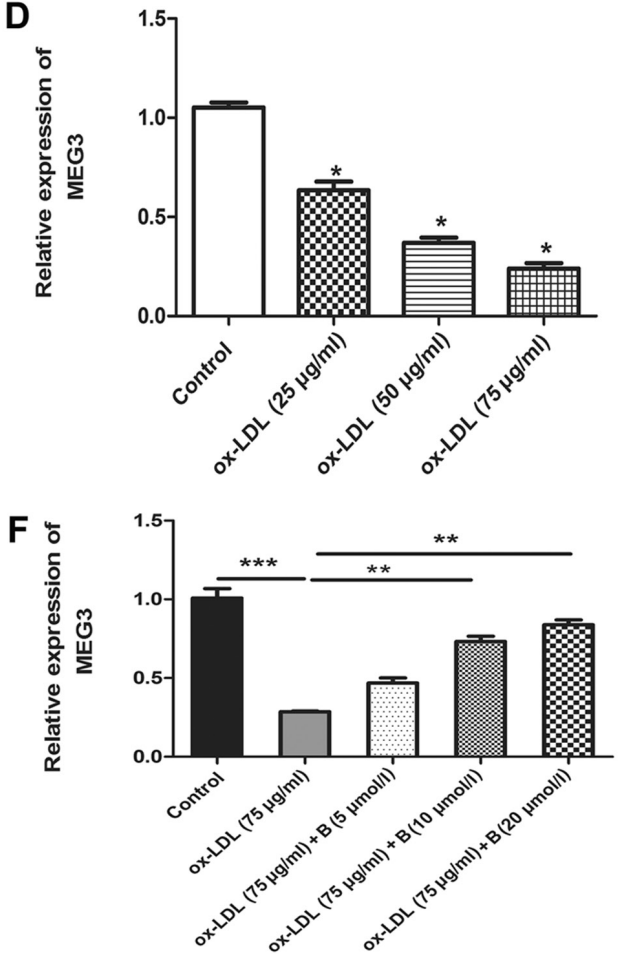

Figure 2. Expression of MEG3 in serum samples and ox-LDL-treated HA-VSMCs, and the effect of treatment with baicalin. (A) The expression of MEG3 in serum samples from patients and controls. $n=40$. ${ }^{*} \mathrm{P}<0.05$, as indicated. (B) MTT assay was performed to determine the HA-VSMC viability following treatment with different concentrations of ox-LDL $(0,25,50$ and $75 \mu \mathrm{g} / \mathrm{ml})$. ${ }^{*} \mathrm{P}<0.05$ vs. the control group. $\mathrm{n}=6$. (C) BrdU assay was performed to determine DNA synthesis following treatment with different concentrations of ox-LDL $(0,25,50$ and $75 \mu \mathrm{g} / \mathrm{ml})$. $\mathrm{n}=6$. $^{*} \mathrm{P}<0.05 \mathrm{vs}$. the control group. (D) $\mathrm{HA}-\mathrm{VSMCs}$ were treated with different concentrations of ox-LDL $(0,25,50$ and $75 \mu \mathrm{g} / \mathrm{ml})$ for $24 \mathrm{~h}$, followed by the detection of MEG3 expression. $\mathrm{n}=6$. ${ }^{*} \mathrm{P}<0.05 \mathrm{vs}$. the control group. (E) Effect of baicalin on MEG3 expression. (F) Effect of baicalin on MEG3 expression in cells treated with ox-LDL. $\mathrm{n}=6$. ${ }^{* *} \mathrm{P}<0.01$ and ${ }^{* * * *} \mathrm{P}<0.001$, as indicated. MEG3, maternally expressed gene 3; B, baicalin; ox-LDL, oxidized low-density lipoprotein; HA-VSMC, human aorta vascular smooth muscle cell; BrdU, bromodeoxyuridine.

compared with the control. Baicalin reversed this effect in a dose-dependent manner (Fig. 2F).

Baicalin inhibits MEG3 silencing-induced proliferation of $o x-L D L$-treated HA-VSMCs. To verify the function of MEG3 in the progression of AS, the current study determined the effects of MEG3 silencing on proliferation of ox-LDL-treated HA-VSMCs. HA-VSMCs treated with $75 \mu \mathrm{g} / \mathrm{ml}$ ox-LDL were transfected with si-MEG3 and treated with different concentration of baicalin $(5,10$ and $20 \mu \mathrm{mol} / \mathrm{l})$, followed by measurement of cell proliferative ability. The knockdown of MEG3 was confirmed by qPCR (Fig. 3A). BrdU assay indicated that downregulation of MEG3 caused a significant increase in cell proliferation compared with the si-NC group in ox-LDL-treated HA-VSMCs. However, cell proliferation was inhibited following treatment with different concentrations of baicalin (Fig. 3B). To determine whether baicalin affected cell cycle progression by increasing MEG3 expression in the presence of ox-LDL, flow cytometry was used to detect the number of cells at different stages of the cell cycle. MEG3 knockdown increased the percentage of cells at the $\mathrm{G}_{2} / \mathrm{M}+\mathrm{S}$ phase compared with the si-NC group. Baicalin inhibited cell cycle progression and increased the number of HA-VSMCs at the $G_{0} / G_{1}$ phase (Fig. $3 C$ and D). EdU staining analysis indicated that the number of proliferating cells increased following knockdown of MEG3 compared with the si-NC group (Fig. 3E and F). This effect was reversed in the presence of baicalin at a concentration of 10 or $20 \mu \mathrm{mol} / \mathrm{l}$. MEG3 is a tumor suppressor gene, and its ectopic expression inhibited cell proliferation and promoted cell apoptosis in human glioma cell line (34). Our previous study indicated that MEG3 knockdown may trigger human pulmonary artery smooth muscle cell 


\section{A}

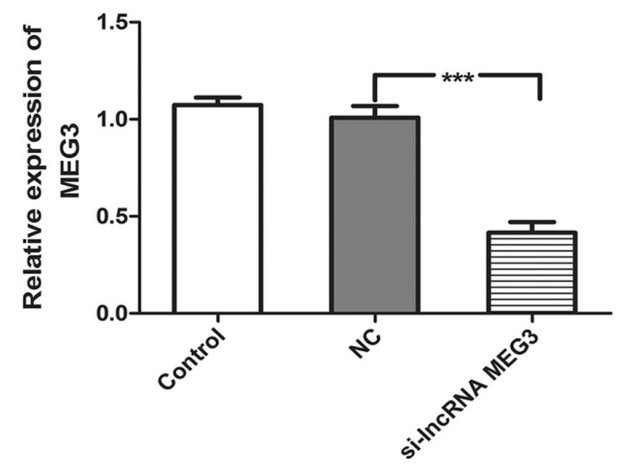

C

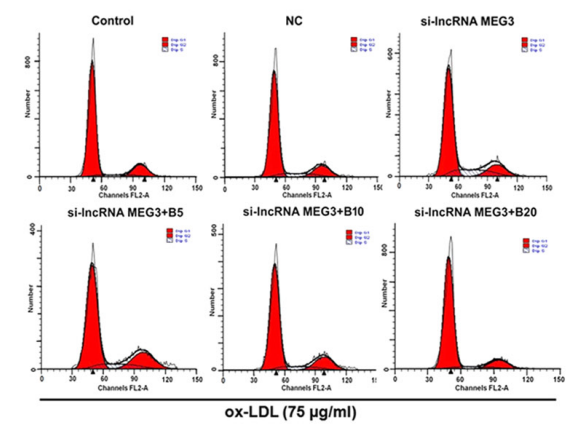

E

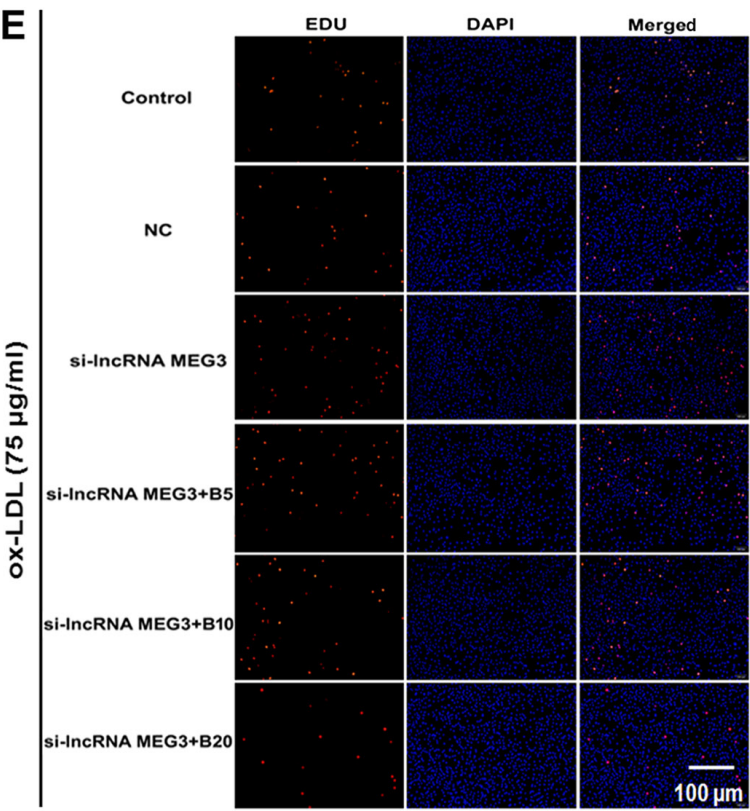

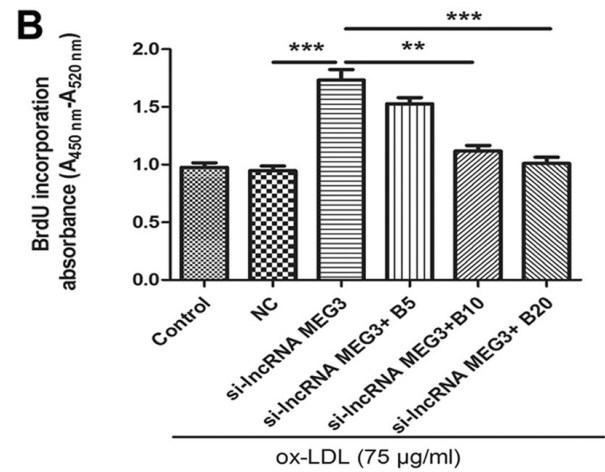

D

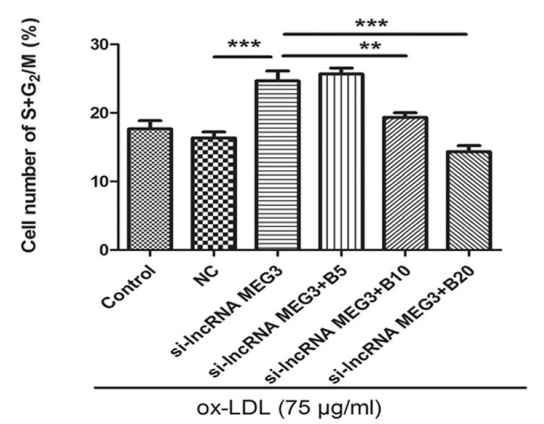

$\mathbf{F}$

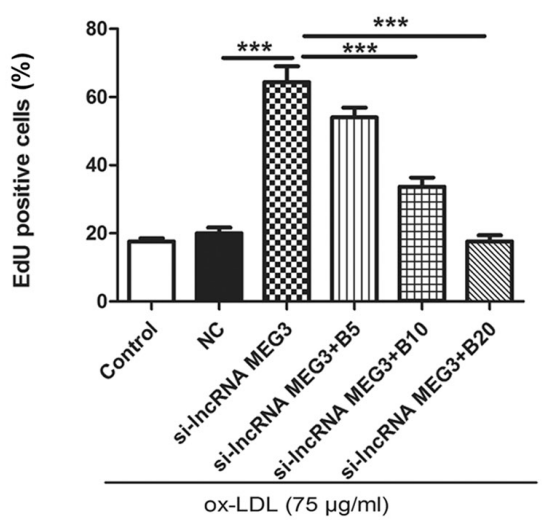

Figure 3. Baicalin reverses MEG3 knockdown-induced proliferation in ox-LDL-treated HA-VSMCs. (A) Reverse transcription-quantitative polymerase chain reaction verified MEG3 knockdown efficiency. (B) BrdU analysis of DNA synthesis. (C) Flow cytometry plots. (D) Quantitative analysis of flow cytometry results on cell cycle progression. (E) EdU staining to study cell proliferation. (F) Quantitative analysis of EdU staining results. $n=6$. ${ }^{* * *} \mathrm{P}<0.01$ and ${ }^{* * * *} \mathrm{P}<0.001$, as indicated. si, small interfering; lnc, long noncoding; MEG3, maternally expressed gene 3; B, baicalin; ox-LDL, oxidized low-density lipoprotein; HA-VSMC, human aorta vascular smooth muscle cell; BrdU, bromodeoxyuridine; $\mathrm{NC}$, negative control; 5, 10 and 20 refer to the concentration of baicalin in $\mu$ mol/l; EdU, 5-ethynyl-2'-deoxyuridine.

proliferation and migration (15). In the present study, MEG3 knockdown promoted the proliferation of HA-VSMCs in the absence of ox-LDL, compared with the si-NC group. However, compared with the MEG3 knockdown group untreated with ox-LDL, proliferation increased in cells transfected with si-lncRNA MEG3 and treated with ox-LDL (Fig. 4A). In the absence of ox-LDL, baicalin inhibited the proliferation of HA-VSMCs transfected with si-lncRNA (Fig. 4B). The above results indicated that baicalin inhibited the proliferation induced by MEG3 knockdown and stimulation with ox-LDL.
Furthermore, RT-qPCR analysis of MEG3 expression was performed following transfection with pCDNA-MEG-3. Compared with the pCDNA group, the expression level of MEG3 increased by 1.59-fold in the pCDNA-MEG3 group (Fig. 5A). Furthermore, overexpression of MEG3 suppressed the viability and proliferation in ox-LDL-treated HA-VSMCs compared with the ox-LDL + pCDNA group (Fig. 5B and C).

Baicalin inhibits MEG3 knockdown-induced migration in ox-LDL-treated HA-VSMCs. It has been reported that 
A

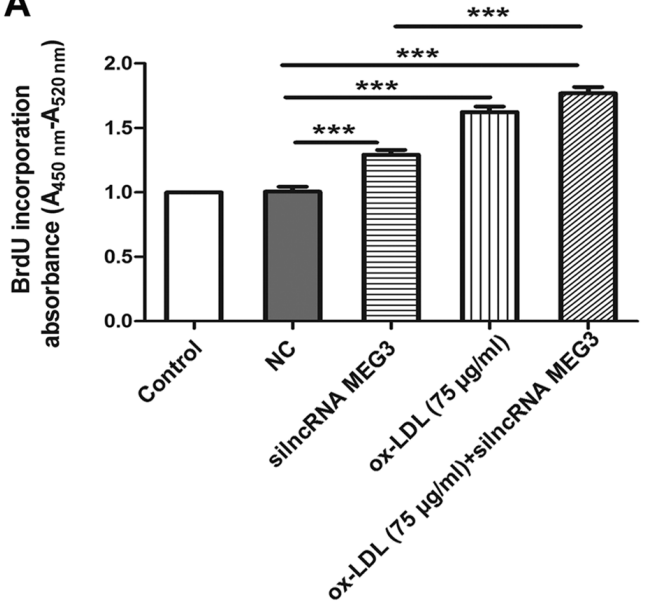

B

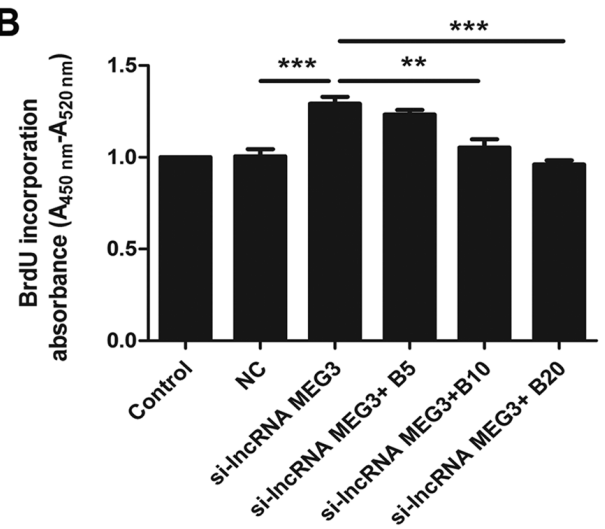

Figure 4. Effect of MEG3 silencing on cell proliferation. (A) BrdU assay was used to analyze the effect of MEG3 knockdown in the proliferation of HA-VSMCs in the absence and presence of ox-LDL. (B) BrdU assay was used to analyze the effect of baicalin on the proliferation of HA-VSMCs in the absence of ox-LDL. ${ }^{* *} \mathrm{P}<0.01$ and ${ }^{* * * *} \mathrm{P}<0.001$, as indicated. $\mathrm{n}=6$. BrdU, bromodeoxyuridine; si, small interfering; lnc, long noncoding; MEG3, maternally expressed gene 3; $\mathrm{B}$, baicalin; ox-LDL, oxidized low-density lipoprotein; HA-VSMC, human aorta vascular smooth muscle cell; NC, negative control; 5, 10 and 20 refer to the concentration of baicalin in $\mu \mathrm{mol} / 1$.
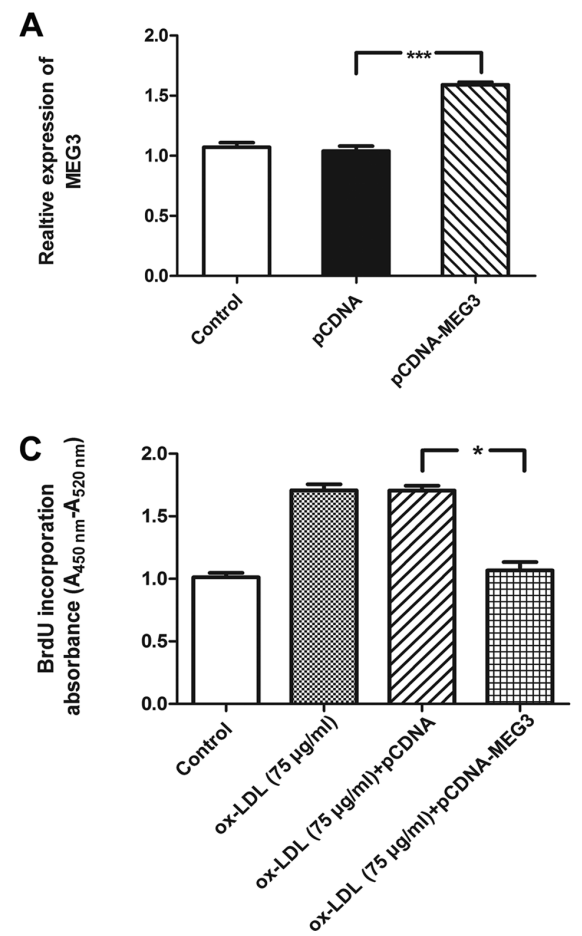

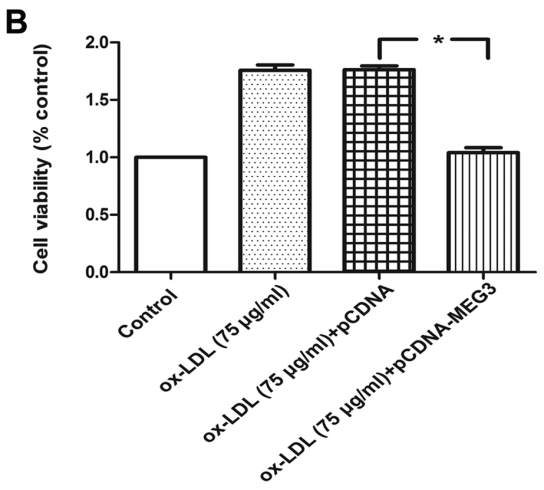

Figure 5. Effect of MEG3 overexpression on ox-LDL-treated HA-VSMCs. (A) MEG3 expression levels following transfection with pCDNA or pCDNA-MEG3. (B) MTT assay was performed to determine the viability cells following overexpression of MEG3 and treatment with ox-LDL in HA-VSMCs. (C) BrdU assay was performed to determine DNA synthesis following overexpression of MEG3 and treatment with ox-LDL in HA-VSMCs. "P<0.05 and ${ }^{* * * *} \mathrm{P}<0.001$, as indicated. $\mathrm{n}=6$. MEG3, maternally expressed gene 3; ox-LDL, oxidized low-density lipoprotein; HA-VSMC, human aorta vascular smooth muscle cell; BrdU, bromodeoxyuridine.

migration of HA-VSMCs contributed to vascular remodeling in AS (35). The current study examined the effect of baicalin on MEG3 silencing-induced migration of HA-VSMCs using a wound healing assay. Ox-LDL- treated HA-VSMCs were treated with control, si-NC, si-lncRNA MEG3, si-lncRNA MEG3 + baicalin (5 $\mu \mathrm{mol} / \mathrm{l})$, si-lncRNA MEG3 + baicalin $(10 \mu \mathrm{mol} / \mathrm{l})$ and si-lncRNA MEG3 + baicalin $(20 \mu \mathrm{mol} / \mathrm{l})$. A scratch was made using a pipette tip and images were captured at 0 and $24 \mathrm{~h}$ to analyze the degree of migration in different treatment groups. The results indicated that knockdown of MEG3 increased the migration of HA-VSMCs, compared with the si-NC group; however, treatment with baicalin at a dose of 10 and $20 \mu \mathrm{mol} / 1$ significantly reversed the effect of MEG3 knockdown (Fig. 6A and B).

Baicalin reverses the effect of MEG3 silencing on cell proliferation and promotes apoptosis in ox-LDL-induced HA-VSMCs. In order to further investigate whether baicalin 

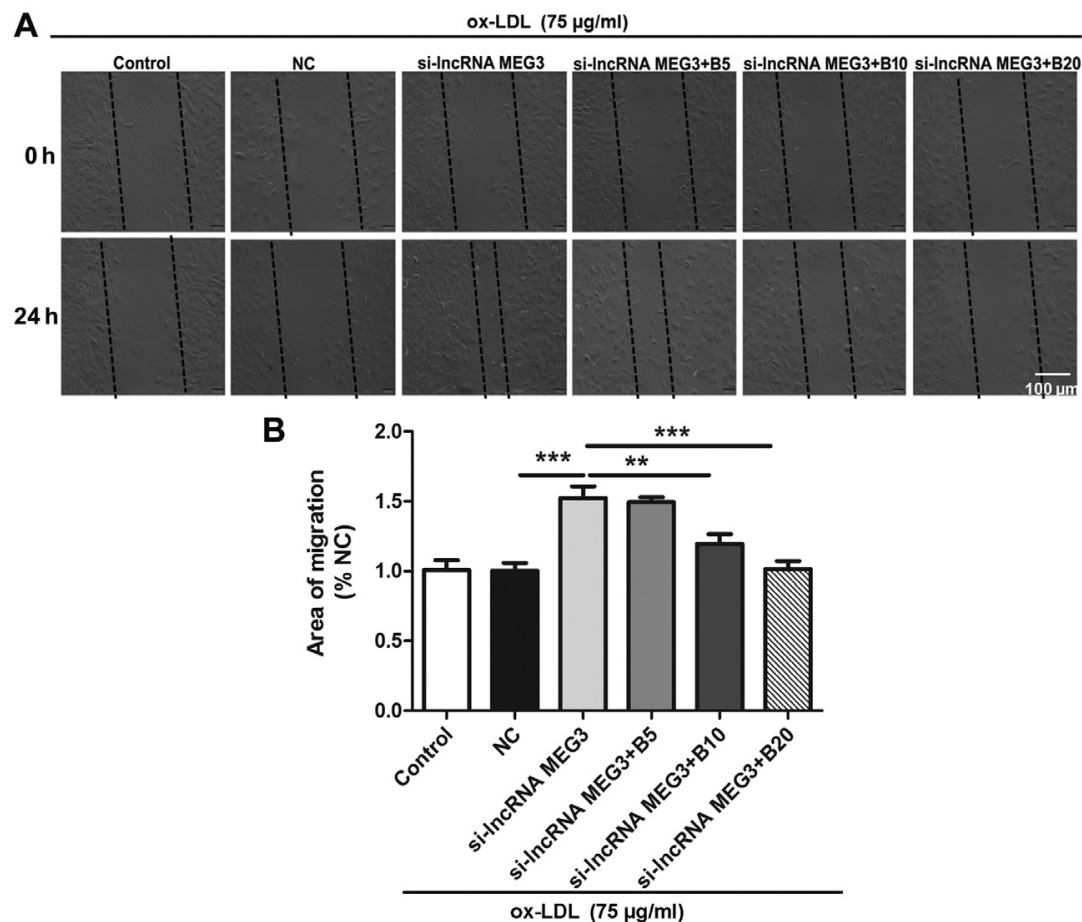

Figure 6. Effect of baicalin on MEG3 knockdown-induced migration in ox-LDL- treated human aorta vascular smooth muscle cells. (A) Wound healing assay. (B) Quantitative analysis of cell migration. $\mathrm{n}=6 .{ }^{* *} \mathrm{P}<0.01$ and ${ }^{* * * *} \mathrm{P}<0.001$, as indicated. NC, negative control; si, small interfering; lnc, long noncoding; MEG3, maternally expressed gene 3; B, baicalin; 5, 10 and 20 refer to the concentration of baicalin in $\mu \mathrm{mol} / 1$.
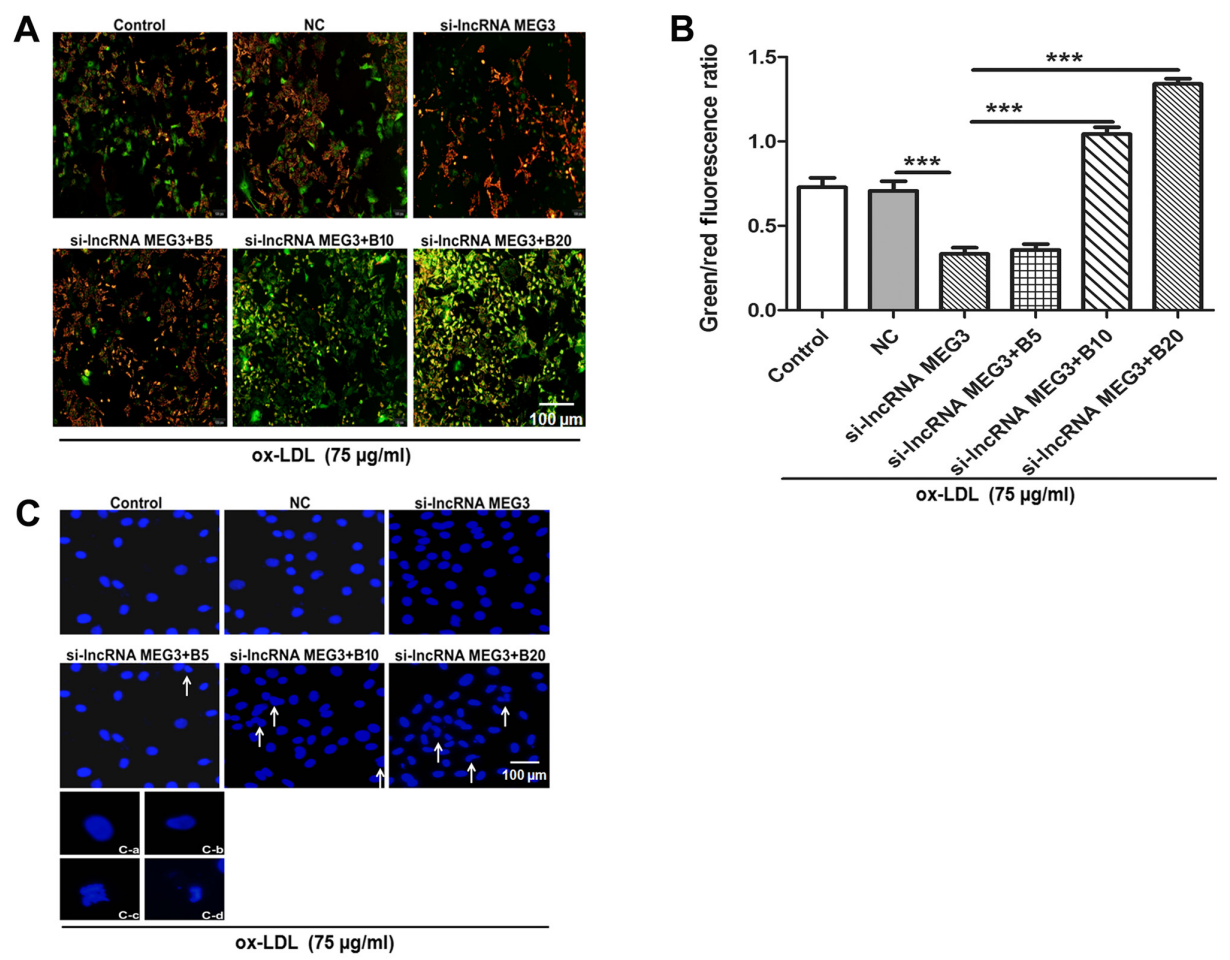

Figure 7. Baicalin reverses the effect of MEG3 silencing on proliferation and promotes apoptosis in ox-LDL-induced HA-VSMCs. (A) JC-1 staining was used to detect apoptosis of human aorta vascular smooth muscle cells. (B) Quantitative analysis of JC-1 staining. (C) Hoechst staining was used to detect human aorta vascular smooth muscle cell apoptosis following treatment with si-lncMEG3 and different concentrations of baicalin. (C-a) A cell with normal nuclear morphology. (C-b) A cell with a crenated nucleus. (C-c) A cell exhibiting nuclear condensation. (C-d) A cell exhibiting nuclear fragmentation. White arrows indicate different cell morphologies. C-a, C-b, C-c and C-d were captured at a magnification of $\mathrm{x} 40 . \mathrm{n}=6 .{ }^{* * * *} \mathrm{P}<0.001$, as indicated. NC, negative control; si, small interfering; lnc, long noncoding; MEG3, maternally expressed gene 3; B, baicalin; 5, 10 and 20 refer to the concentration of baicalin in $\mu$ mol/1.

could promote apoptosis in AS in vitro, the expression of MEG3 was decreased in HA-VSMCs through pre-treatment with ox-LDL. Alterations in mitochondrial function were initially investigated. According to previous studies, disruption 

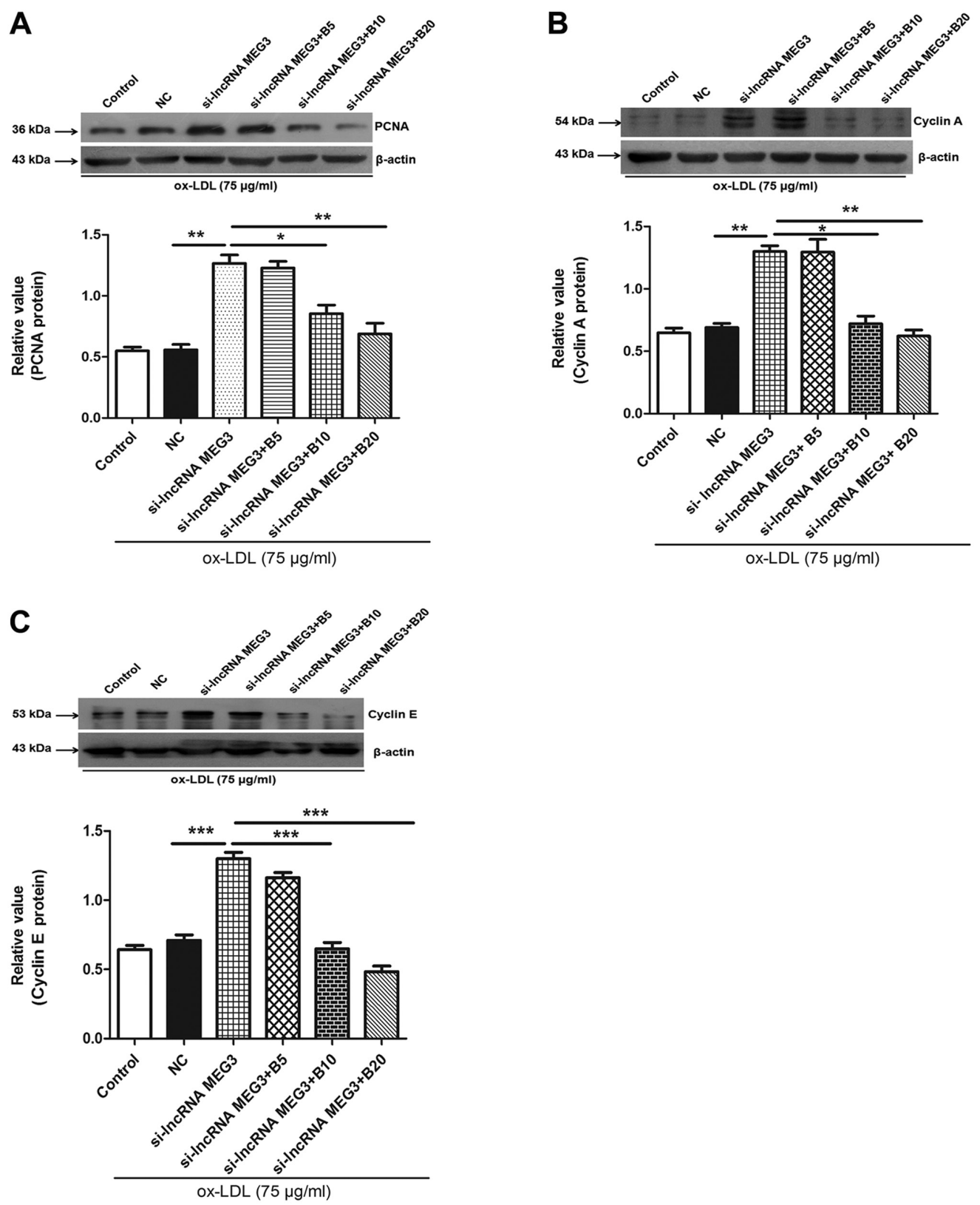

Figure 8. Effect of baicalin on the proliferation of ox-LDL-treated human aorta vascular smooth muscle cells. Expression levels of (A) PCNA (B) cyclin A and (C) cyclin E. $\beta$-actin was used as the loading control. $\mathrm{n}=4 .{ }^{*} \mathrm{P}<0.05,{ }^{* * *} \mathrm{P}<0.01$ and ${ }^{* * * *} \mathrm{P}<0.001$, as indicated. NC, negative control; si, small interfering; lnc, long noncoding; MEG3, maternally expressed gene 3; B, baicalin; 5, 10 and 20 refer to the concentration of baicalin in $\mu$ mol/1; PCNA, proliferating cell nuclear antigen.

of mitochondrial membrane potential is an early event of apoptosis $(31,36,37)$. The alterations of mitochondrial membrane potential were verified by JC-1 staining. Following MEG3 knockdown the green/red fluorescence ratio was reduced compared with the si-NC group, suggesting an increase in mitochondrial membrane potential. By contrast, the mitochondrial membrane potential decreased following treatment with baicalin, as evidenced by increased ratio of green to red fluorescence (Fig. 7A and B). Furthermore, chromatin condensation and cell nuclear morphology were observed by staining with Hoechst 33342. The MEG3 knockdown group exhibited the same complete nuclear morphology as the NC group in cells, while treatment with baicalin resulted in alterations in chromatin morphology including crenation, condensation and fragmentation (Fig. 7C).
Baicalin inhibits the expression of PCNA, cyclin A and E in $H A$-VSMCs. To further determine the mechanism underlying baicalin-mediated inhibition of MEG3 knockdown-induced proliferation, the expression of PCNA was determined by western blotting. The results indicated that MEG3 knockdown increased the expression of PCNA, which was reversed in the presence of different concentration of baicalin (Fig. 8A). Cyclin A and E serve roles in cell cycle progression, and, therefore, expression levels of these proteins were determined in ox-LDL-stimulated HA-VSMCs. The results indicated that knockdown of MEG3 increased the expression of cyclin A and E; however, this effect was reversed by treatment with baicalin (Fig. 8B and C). These results indicated that baicalin may serve a role in regulating cell cycle progression and inhibiting cell proliferation. 
A ox-LDL $(75 \mu \mathrm{g} / \mathrm{ml})$
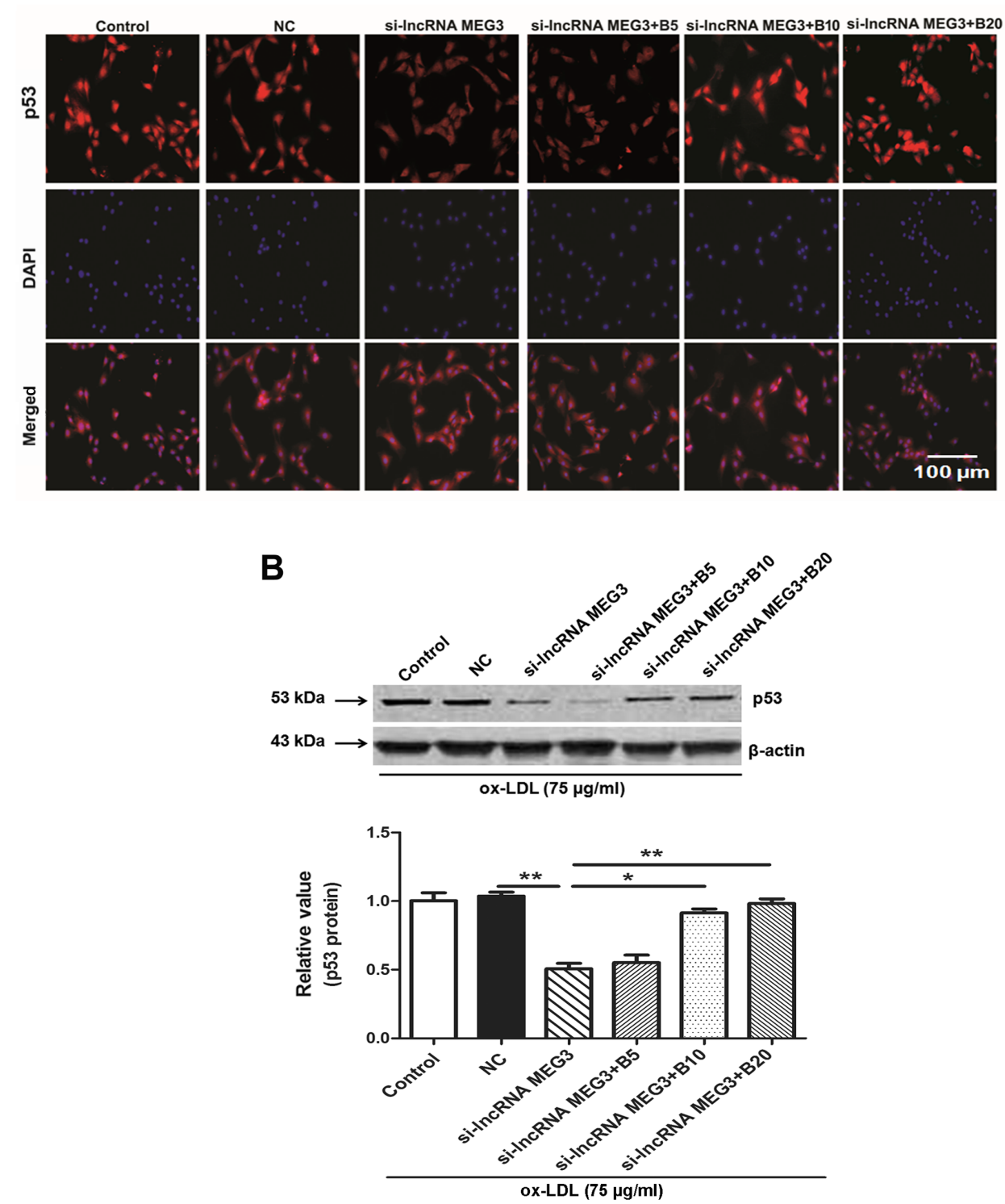

Figure 9. Effect of baicalin on the ox-LDL-stimulated cell growth via the MEG3/p53 pathway in human aorta vascular smooth muscle cells. (A) Immunofluorescence assay of p53 translocation. Red color indicates p53-positive staining and blue color indicates DAPI staining. (B) Western blot analysis of 53 protein expression. $\beta$-actin was used as the loading control. $\mathrm{n}=4$. ${ }^{*} \mathrm{P}<0.05$ and ${ }^{* *} \mathrm{P}<0.01$, as indicated. $\mathrm{NC}$, negative control; si, small interfering; lnc, long noncoding; MEG3, maternally expressed gene 3; B, baicalin; 5, 10 and 20 refer to the concentration of baicalin in $\mu \mathrm{mol} / 1$.

Baicalin suppresses the ox-LDL-stimulated cell growth via modulation of the MEG3/p53 pathway in HA-VSMCs. Previous studies demonstrated that the p53 signaling pathway serves a role in AS $(38,39)$. To further examine the mechanism underling baicalin-mediated inhibition of cell growth, p53 immunofluorescence and western blotting were performed in ox-LDL-treated HA-VSMCs. Fluorescence data indicated that p53 was expressed in the nucleus under normal conditions; however, cytoplasmic expression was detected following knockdown of MEG3. Following treatment with different concentrations of baicalin, p53 was activated and returned to nuclear expression (Fig. 9A). Western blotting further indicated that the low expression of p53 following MEG3 knockdown gradually increased following administration of different concentrations of baicalin (Fig. 9B). The above data indicated that baicalin inhibited the growth of HA-VSMCs possibly through the p53 signaling pathway.

\section{Discussion}

At present, AS is one of the most common cardiovascular disorders, even though there have been significant improvements in clinical diagnosis and control of symptoms $(2,7,40)$. AS remains a major health concern and exhibits high morbidity and mortality rates worldwide (41). A number of studies indicated that lncRNAs serve important roles in the progression of AS $(2,27,42)$. The current study demonstrated that lncRNA MEG3 may be an important regulator of cell proliferation and apoptosis in vitro. MEG3 knockdown promoted the proliferation of HA-VSMCs in the absence of ox-LDL. Furthermore, in the presence of ox-LDL, MEG3 knockdown increased the proliferation even further. Ox-LDL can induce AS via multiple mechanisms, including promotion of EC activation and dysfunction, VSMC proliferation and migration, and foam cell formation $(32,43)$. VSMCs, the major cell type in blood 
vessels, participate in remodeling of the arterial wall in order to maintain blood flow in vessels affected by AS $(3,44)$. MEG3 is a tumor suppressor gene and its ectopic expression inhibited cell proliferation and promoted cell apoptosis in human glioma cell line(34). Therefore, the present study aimed to investigate whether MEG3 was involved in the regulation of AS progression in ox-LDL-stimulated HA-VSMCs. The results indicated that the expression of MEG3 was reduced in serum samples from patients with AS and ox-LDL-induced HA-VSMCs, compared with normal control samples and cells untreated with ox-LDL, respectively. Further experiments indicated that the decrease in MEG3 expression was reversed by treatment with baicalin in a concentration-dependent manner. Knockdown of endogenous MEG3 promoted proliferation and migration, and inhibited apoptosis in HA-VSMCs. Baicalin reversed the effects of MEG3 knockdown on proliferation, migration and apoptosis in ox-LDL-treated HA-VSMCs. We previously reported that MEG3 knockdown triggered human pulmonary artery smooth muscle cell proliferation and migration (15). The current study demonstrated increased expression levels of cyclin A and E following MEG3 knockdown. However, following treatment of HA-VSMCs with different concentrations of baicalin, the MEG3 knockdown-induced effect on the expression levels of cyclin A and $\mathrm{E}$ was reversed. Baicalin inhibited proliferation and facilitated apoptosis through activating the MEG3/p53 expression. This result suggests a regulatory activity of IncRNA MEG3 in AS and this molecule may be a novel therapeutic target for the treatment of human cardiovascular disease.

Abnormal proliferation, apoptosis and migration of VSMCs are considered key factors in the development of AS (4,45). Previous studies have demonstrated that lncRNAs serve important roles in the development of AS $(2,27,46)$. Numerous studies demonstrated the potential role of lncRNAs in proliferation and migration of smooth muscle cells. A recent study suggested that lncRNA-H19 knockdown inhibited proliferation and induced apoptosis of HA-VSMCs by inactivating microRNA-148b/WNT/ $\beta$-catenin signaling pathway, suggesting the potential application of $\mathrm{H} 19$ in the prevention of AS (27), which could be used as a potential target for AS. Another study reported that lncRNA antisense RNA in the INK4 locus (ANRIL) was closely associated with the severity of AS (46). ANRIL increased the proliferation of VSMCs by reducing the expression of cyclin dependent kinase inhibitor 2A and B (47). LincRNA-p21, a novel regulatory factor, has been revealed to regulate neointima formation, proliferation, apoptosis and AS in VSMCs by increasing the activity of p53 (2). To the best of our knowledge, the current study is the first to identify an interaction between MEG3 and AS in vitro. In serum samples from patients with AS, the expression of MEG3 was significantly reduced compared with the normal control group. Furthermore, silencing of MEG3 significantly accelerated proliferation and migration, and inhibited apoptosis of HA-VSMCs, suggesting that MEG3 silencing may suppress AS progression.

Baicalin has been reported to induce anti-inflammatory (21) and antiproliferative effects (17). Baicalin is widely used in the treatment of inflammatory diseases $(21,48)$ and dysfunction of VSMCs and ECs $(17,49)$. Numerous studies have confirmed that baicalin serves anti-atherosclerotic roles by inhibiting inflammation (21) and reducing oxidative stress (19). In addition, baicalin can protect chronic hypoxia-induced pulmonary hypertension and cor pulmonale by reducing the activity of the p38 mitogen-activated protein kinase pathway and matrix matalloprotease-9 (50). In a previous study, baicalin and geniposide inhibited the progression of AS by enhancing the expression of Wnt family member 1 and attenuating the expression of dickkopf-related protein 1 (51). In a high-cholesterol diet, baicalin decreased the expression of monocyte chemoattractant protein 1 and interleukin- 6 in the kidneys of ApoE knock out mice (23). Although baicalin has been demonstrated to exhibit a number of biological functions, the underlying mechanism of baicalin-mediated prevention of AS progression by altering lncRNA expression is not well documented. The present study indicated that baicalin at a dose of 10 and $20 \mu \mathrm{mol} / 1$ significantly inhibited the proliferation and migration, and promoted apoptosis of HA-VSMCs treated with xo-LDL. The antiproliferative effect of baicalin was associated with cell cycle arrest at the $\mathrm{G}_{0} / \mathrm{G}_{1}$ stage. Cell cycle progression is regulated by a complex regulatory molecular network, including cyclins (52). Cyclin A and $\mathrm{E}$ are the main mediators during the cell cycle progression from the $G_{0} / G_{1}$ to the $\mathrm{S}$ phase (53). In the present study, baicalin arrested the cell cycle at the $G_{0} / G_{1}$ phase and decreased the expression of cell cycle regulatory proteins PCNA, cyclin A and E in ox-LDL-treated HA-VSMCs with MEG3 knockdown. It may be hypothesized that baicalin inhibited cell proliferation via downregulation of expression of cell cycle regulatory proteins, which resulted in cell cycle arrest at the $G_{0} / G_{1}$ phase.

The p53 signaling pathway has been reported to be involved in vascular remodeling and atherosclerotic plaque formation $(15,54,55)$. In a previous study, the expression of p53 in atherosclerotic plaques was negatively correlated with cell proliferation (56). In the rabbit carotid artery, overexpression of p53 significantly inhibited cell proliferation (57). In addition, high expression of p53 may inhibit monocyte/macrophage cell division and promote the apoptosis of macrophages, thus preventing the development of AS (25). The current study confirmed that baicalin may increase the expression of p53 and promote its transport from the cytoplasm to nucleus following MEG3 silencing. These results suggest that baicalin may ameliorate AS and the MEG3/p53 signaling pathway may be involved in this process.

In conclusion, baicalin inhibited proliferation and promoted apoptosis in ox-LDL-treated HA-VSMCs by activating the expression of MEG3/p53. Baicalin may be used to increase the expression of MEG3/p53 and prevent AS. However, in future clinical trials, the administration of baicalin to patients with AS may provide understanding of disease pathways and further clarify the therapeutic potential of baicalin.

\section{Acknowledgements}

Not applicable.

\section{Funding}

The study is supported by the Open Fund of Key Laboratory of Minstry of Education for TCM Viscera State Theory and Applications, Liaoning University of Traditional Chinese 
Medicine (grant no. zyzx1605), National Natural Science Foundation of China (grant no. 31871155), Natural Science Foundation of Jiangsu Province (grant no. BK20161297), the Social Fund for Development of Lianyungang Science and Technology Bureau (grant no. SH1612), project of Jiangsu Provincial Commission of Health and Family Planning (grant no. QNRC2016505), and Six Big Talent Peak C Project (grant no. YY-110).

\section{Availability of data and materials}

All data generated or analysed during this study are included in this published article.

\section{Authors' contributions}

YL designed the present study and the experiments, and wrote the manuscript. LJ conducted western blotting and RT-qPCR experiments. DM performed BrdU, EdU, JC-1 and flow cytometry assays. YX performed cell culture, cell transfection and wound healing assay. JZ performed patient blood sample collection and Hoechst staining. ZS performed immunofluorescence assay. All authors read and approved the final version of the manuscript.

\section{Ethics approval and consent to participate}

The present study was approved by the Ethics Committee for the use of human samples of the First People's Hospital of Lianyungang, which was in accordance with the code of ethics of the Declaration of Helsinki developed by the World Medical Association. Each individual provided written informed consent prior to their participation.

\section{Patient consent for publication}

Not applicable.

\section{Competing interests}

The authors declare that they have no competing interests.

\section{References}

1. Antoniades C, Antonopoulos AS, Bendall JK and Channon KM: Targeting redox signaling in the vascular wall: from basic science to clinical practice. Curr Pharm Des 15: 329-342, 2009.

2. Wu G, Cai J, Han Y, Chen J, Huang ZP, Chen C, Cai Y, Huang H, Yang Y, Liu Y, et al: LincRNA-p21 regulates neointima formation, vascular smooth muscle cell proliferation, apoptosis, and atherosclerosis by enhancing p53 activity. Circulation 130: 1452-1465, 2014.

3. Chistiakov DA, Orekhov AN and Bobryshev YV: Vascular smooth muscle cell in atherosclerosis. Acta Physiol (Oxf) 214: 33-50, 2015.

4. Doran AC, Meller N and McNamara CA: Role of smooth muscle cells in the initiation and early progression of atherosclerosis. Arterioscler Thromb Vasc Biol 28: 812-819, 2008.

5. Ponting CP, Oliver PL and Reik W: Evolution and functions of long noncoding RNAs. Cell 136: 629-641, 2009.

6. Zhao XY and Lin JD: Long Noncoding RNAs: a new regulatory code in metabolic control. Trends Biochem Sci 40: 586-596, 2015

7. Liu Y, Zheng L, Wang Q and Hu YW: Emerging roles and mechanisms of long noncoding RNAs in atherosclerosis. Int J Cardiol 228: 570-582, 2017.
8. Ballantyne MD, Pinel K, Dakin R, Vesey AT, Diver L, Mackenzie R, Garcia R, Welsh P, Sattar N, Hamilton G, et al: Smooth muscle enriched long noncoding RNA (SMILR) regulates cell proliferation. Circulation 133: 2050-2065, 2016.

9. Liu JY, Yao J, Li XM, Song YC, Wang XQ, Li YJ, Yan B and Jiang Q: Pathogenic role of lncRNA-MALAT1 in endothelial cell dysfunction in diabetes mellitus. Cell Death Dis 5: e1506, 2014.

10. Michalik KM, You X, Manavski Y, Doddaballapur A, Zörnig M, Braun T, John D, Ponomareva Y, Chen W, Uchida S, et al: Long noncoding RNA MALAT1 regulates endothelial cell function and vessel growth. Circ Res 114: 1389-1397, 2014.

11. Shan K, Jiang Q, Wang XQ, Wang YN, Yang H, Yao MD, Liu C, $\mathrm{Li}$ XM, Yao J, Liu B, et al: Role of long non-coding RNA-RNCR3 in atherosclerosis-related vascular dysfunction. Cell Death Dis 7: e2248, 2016.

12. Lu KH, Li W, Liu XH, Sun M, Zhang ML, Wu WQ, Xie WP and Hou YY: Long non-coding RNA MEG3 inhibits NSCLC cells proliferation and induces apoptosis by affecting p53 expression. BMC Cancer 13: 461, 2013.

13. Cui HB, Ge HE, Wang YS and Bai XY: MiR-208a enhances cell proliferation and invasion of gastric cancer by targeting SFRP1 and negatively regulating MEG3. Int J Biochem Cell Biol 102: 31-39, 2018

14. Zheng Q, Lin Z, Xu J, Lu Y, Meng Q, Wang C, Yang Y, Xin X, Li X, Pu H, et al: Long noncoding RNA MEG3 suppresses liver cancer cells growth through inhibiting $\beta$-catenin by activating PKM2 and inactivating PTEN. Cell Death Dis 9: 253, 2018.

15. Sun Z, Nie X, Sun S, Dong S, Yuan C, Li Y, Xiao B, Jie D and Liu Y: Long non-coding RNA MEG3 downregulation triggers human pulmonary artery smooth muscle cell proliferation and migration via the p53 signaling pathway. Cell Physiol Biochem 42: 2569-2581, 2017.

16. Li C, Lin G and Zuo Z: Pharmacological effects and pharmacokinetics properties of Radix Scutellariae and its bioactive flavones. Biopharm Drug Dispos 32: 427-445, 2011.

17. Dong LH, Wen JK, Miao SB, Jia Z, Hu HJ, Sun RH, Wu Y and Han M: Baicalin inhibits PDGF-BB-stimulated vascular smooth muscle cell proliferation through suppressing PDGFR $\beta$-ERK signaling and increase in p27 accumulation and prevents injuryinduced neointimal hyperplasia. Cell Res 20: 1252-1262, 2010.

18. Zhu W, Jin Z, Yu J, Liang J, Yang Q, Li F, Shi X, Zhu X and Zhang X: Baicalin ameliorates experimental inflammatory bowel disease through polarization of macrophages to an M2 phenotype. Int Immunopharmacol 35: 119-126, 2016.

19. Waisundara VY, Siu SY, Hsu A, Huang D and Tan BK: Baicalin upregulates the genetic expression of antioxidant enzymes in type-2 diabetic Goto-Kakizaki rats. Life Sci 88: 1016-1025, 2011.

20. Wang Z, Ma L, Su M, Zhou Y, Mao K, Li C, Peng G, Zhou C, Shen B and Dou J: Baicalin induces cellular senescence in human colon cancer cells via upregulation of DEPP and the activation of Ras/Raf/MEK/ERK signaling. Cell Death Dis 9: 217, 2018.

21. Yang X, Zhang Q, Gao Z, Yu C and Zhang L: Baicalin alleviates IL-1 $\beta$-induced inflammatory injury via down-regulating miR-126 in chondrocytes. Biomed Pharmacother 99: 184-190, 2018.

22. Ku SK and Bae JS: Baicalin, baicalein and wogonin inhibits high glucose-induced vascular inflammation in vitro and in vivo. BMB Rep 48: 519-524, 2015.

23. Liu L, Liao P, Wang B, Fang X, Li W and Guan S: Baicalin inhibits the expression of monocyte chemoattractant protein-1 and interleukin-6 in the kidneys of apolipoprotein E-knockout mice fed a high cholesterol diet. Mol Med Rep 11: 3976-3980, 2015.

24. Guevara NV, Kim HS, Antonova EI and Chan L: The absence of p53 accelerates atherosclerosis by increasing cell proliferation in vivo. Nat Med 5: 335-339, 1999.

25. Mercer J, Figg N, Stoneman V, Braganza D and Bennett MR: Endogenous p53 protects vascular smooth muscle cells from apoptosis and reduces atherosclerosis in ApoE knockout mice. Circ Res 96: 667-674, 2005.

26. el-Deiry WS, Tokino T, Velculescu VE, Levy DB, Parsons R, Trent JM, Lin D, Mercer WE, Kinzler KW and Vogelstein B: WAF1, a potential mediator of p53 tumor suppression. Cell 75: 817-825, 1993

27. Zhang L, Cheng H, Yue Y, Li S, Zhang D and He R: H19 knockdown suppresses proliferation and induces apoptosis by regulating $\mathrm{miR}-148 \mathrm{~b} / \mathrm{WNT} / \beta$-catenin in ox-LDL -stimulated vascular smooth muscle cells. J Biomed Sci 25: 11, 2018.

28. Liu Y, Ma C, Zhang Q, Yu L, Ma J, Zhang L, Hao X, Cao F, Wang $L$ and $Z$ hu D: The key role of transforming growth factor-beta receptor I and 15-lipoxygenase in hypoxia-induced proliferation of pulmonary artery smooth muscle cells. Int J Biochem Cell Biol 44: 1184-1202, 2012. 
29. Livak KJ and Schmittgen TD: Analysis of relative gene expression data using real-time quantitative PCR and the 2(-Delta Delta C(T)) Method. Methods 25: 402-408, 2001.

30. Liu Y, Cao Y, Sun S, Zhu J, Gao S, Pang J, Zhu D and Sun Z: Transforming growth factor-betal upregulation triggers pulmonary artery smooth muscle cell proliferation and apoptosis imbalance in rats with hypoxic pulmonary hypertension via the PTEN/AKT pathways. Int J Biochem Cell Biol 77 (Pt A): 141-154, 2016.

31. Ma J, Liang S, Wang Z, Zhang L, Jiang J, Zheng J, Yu L, Zheng X, Wang $R$ and Zhu D: ROCK pathway participates in the processes that 15-hydroxyeicosatetraenoic acid (15-HETE) mediated the pulmonary vascular remodeling induced by hypoxia in rat. J Cell Physiol 222: 82-94, 2010.

32. Pirillo A, Norata GD and Catapano AL: LOX-1, OxLDL, and atherosclerosis. Mediators Inflamm 2013: 152786, 2013.

33. Tang Y, Xu Q, Peng H, Liu Z, Yang T, Yu Z, Cheng G, Li X, Zhang $G$ and Shi $R$ : The role of vascular peroxidase 1 in ox-LDL-induced vascular smooth muscle cell calcification. Atherosclerosis 243: 357-363, 2015.

34. Wang P, Ren Z and Sun P: Overexpression of the long non-coding RNA MEG3 impairs in vitro glioma cell proliferation. J Cell Biochem 113: 1868-1874, 2012

35. Goncharova EA, Ammit AJ, Irani C, Carroll RG, Eszterhas AJ, Panettieri RA and Krymskaya VP: PI3K is required for proliferation and migration of human pulmonary vascular smooth muscle cells. Am J Physiol Lung Cell Mol Physiol 283: L354-L363, 2002.

36. Salido M, Gonzalez JL and Vilches J: Loss of mitochondrial membrane potential is inhibited by bombesin in etoposide-induced apoptosis in PC-3 prostate carcinoma cells. Mol Cancer Ther 6 : 1292-1299, 2007.

37. Wang Z, Tang X, Li Y, Leu C, Guo L, Zheng X and Zhu D: 20-Hydroxyeicosatetraenoic acid inhibits the apoptotic responses in pulmonary artery smooth muscle cells. Eur J Pharmacol 588: 9-17, 2008

38. Xiong Y, Yu Y, Montani JP, Yang Z and Ming XF: Arginase-II induces vascular smooth muscle cell senescence and apoptosis through p66Shc and p53 independently of its 1-arginine ureahydrolase activity: implications for atherosclerotic plaque vulnerability. J Am Heart Assoc 2: e000096, 2013.

39. Leeper NJ, Raiesdana A, Kojima Y, Kundu RK, Cheng H, Maegdefessel L, Toh R, Ahn GO, Ali ZA, Anderson DR, et al Loss of CDKN2B promotes p53-dependent smooth muscle cell apoptosis and aneurysm formation. Arterioscler Thromb Vasc Biol 33: e1-e10, 2013.

40. Li W, Huang H, Li L, Wang L, Li Y, Wang Y, Guo S, Li L, Wang D, He Y, et al: The pathogenesis of atherosclerosis based on human signaling networks and stem cell expression data. Int J Biol Sci 14: 1678-1685, 2018.

41. Mozaffarian D, Benjamin EJ, Go AS, Arnett DK, Blaha MJ, Cushman M, Das SR, de Ferranti S, Després JP, Fullerton HJ, et al; Writing Group Members; American Heart Association Statistics Committee; Stroke Statistics Subcommittee: Heart disease and stroke statistics-2016 update: a report from the american heart association. Circulation 133: e38-e360, 2016.

42. Li H, Zhu H and Ge J: Long noncoding RNA: recent updates in atherosclerosis. Int J Biol Sci 12: 898-910, 2016.

43. Nakajima K, Nakano T and Tanaka A: The oxidative modification hypothesis of atherosclerosis: the comparison of atherogenic effects on oxidized LDL and remnant lipoproteins in plasma. Clin Chim Acta 367: 36-47.

44. Johnson JL: Emerging regulators of vascular smooth muscle cell function in the development and progression of atherosclerosis. Cardiovasc Res 103: 452-460, 2014
45. Wang P, Xu TY, Guan YF, Zhao Y, Li ZY, Lan XH, Wang X, Yang PY, Kang ZM, Vanhoutte PM, et al: Vascular smooth muscle cell apoptosis is an early trigger for hypothyroid atherosclerosis. Cardiovasc Res 102: 448-459, 2014.

46. Holdt LM, Beutner F, Scholz M, Gielen S, Gäbel G, Bergert H, Schuler G, Thiery $\mathbf{J}$ and Teupser D: ANRIL expression is associated with atherosclerosis risk at chromosome $9 \mathrm{p} 21$. Arterioscler Thromb Vasc Biol 30: 620-627, 2010.

47. Congrains A, Kamide K, Oguro R, Yasuda O, Miyata K, Yamamoto E, Kawai T, Kusunoki H, Yamamoto H, Takeya Y, et al: Genetic variants at the 9p21 locus contribute to atherosclerosis through modulation of ANRIL and CDKN2A/B. Atherosclerosis 220: 449-455, 2012.

48. Hou J, Wang J, Zhang P, Li D, Zhang C, Zhao H, Fu J, Wang B and Liu J: Baicalin attenuates proinflammatory cytokine production in oxygen-glucose deprived challenged rat microglial cells by inhibiting TLR4 signaling pathway. Int Immunopharmacol 14: 749-757, 2012.

49. Kim DH, Cho KH, Moon SK, Kim YS, Kim DH, Choi JS and Chung HY: Cytoprotective mechanism of baicalin against endothelial cell damage by peroxynitrite. J Pharm Pharmacol 57: 1581-1590, 2005.

50. Yan S, Wang Y, Liu P, Chen A, Chen M, Yao D, Xu X, Wang L and Huang X: Baicalin attenuates hypoxia-induced pulmonary arterial hypertension to improve hypoxic cor pulmonale by reducing the activity of the p38 MAPK signaling pathway and MMP-9. Evid Based Complement Alternat Med 2016: 2546402, 2016.

51. Wang B, Liao PP, Liu LH, Fang X, Li W and Guan SM: Baicalin and geniposide inhibit the development of atherosclerosis by increasing Wnt1 and inhibiting dickkopf-related protein-1 expression. J Geriatr Cardiol 13: 846-854, 2016.

52. Bendris N, Lemmers B and Blanchard JM: Cell cycle, cytoskeleton dynamics and beyond: The many functions of cyclins and CDK inhibitors. Cell Cycle 14: 1786-1798, 2015.

53. Braun-Dullaeus RC, Mann MJ, Sedding DG, Sherwood SW, von der Leyen HE and Dzau VJ: Cell cycle-dependent regulation of smooth muscle cell activation. Arterioscler Thromb Vasc Biol 24: 845-850, 2004

54. Mayr U, Mayr M, Li C, Wernig F, Dietrich H, Hu Y and Xu Q: Loss of p53 accelerates neointimal lesions of vein bypass grafts in mice. Circ Res 90: 197-204, 2002.

55. Sanz-González SM, Barquín L, García-Cao I, Roque M, González JM, Fuster JJ, Castells MT, Flores JM, Serrano M and Andrés V: Increased p53 gene dosage reduces neointimal thickening induced by mechanical injury but has no effect on native atherosclerosis. Cardiovasc Res 75: 803-812, 2007.

56. Ihling C, Menzel G, Wellens E, Mönting JS, Schaefer HE and Zeiher AM: Topographical association between the cyclin-dependent kinases inhibitor P21, p53 accumulation, and cellular proliferation in human atherosclerotic tissue. Arterioscler Thromb Vasc Biol 17: 2218-2224, 1997.

57. Scheinman M, Ascher E, Levi GS, Hingorani A, Shirazian D and Seth P: p53 gene transfer to the injured rat carotid artery decreases neointimal formation. J Vasc Surg 29: 360-369, 1999.

This work is licensed under a Creative Commons Attribution-NonCommercial-NoDerivatives 4.0 International (CC BY-NC-ND 4.0) License. 